\title{
Die Massen-Migration aus der SBZ und der DDR
}

\section{H. Reinhard Koch}

Forschungsstelle für Jugendfragen, Hindenburgstraße 13, D-3000 Hannover 1

\section{Anlässe}

Seit einem Vierteljahrhundert ist die Massen-Migration aus der DDR in die Bundesrepublik abgeschlossen; sie hat längst ihre allgemein-politische Bedeutung ${ }^{1}$ verloren; lange Jahre schienen die Ereignisse ausschließlich von zeithistorischem Interesse. Erst in jüngster Zeit hat die Migration der 50er Jahre erneut Aufmerksamkeit erregt.

Im Zusammenhang mit den Gastarbeiterproblemen in der Bundesrepublik sind mehrere demographische Studien über die ökonomisch bedingten, langfristigen Wanderungstendenzen in Deutschland während der letzten 100 bzw. 200 Jahre erschienen, u. a. von Bethlehem (1982), Bade (1983), Köllmann (1983), Marschalk (1984). Dabei waren Ost-West-Wanderungen von besonderem Interesse, in deren Kontext auch die Migration aus der SBZ bzw. der DDR gesehen wurde. Der zweite Anlaß für die erneute Beschäftigung mit der Massen-Migration der 50er Jahre ist die Ausreise„welle“ des Jahres 1984, und die heftigen Reaktionen auf dieses Ereignis. Zum Thema „Flucht“ oder „Flüchtlinge“ kann noch fast jeder Bundesbürger "Selbsterlebtes“ aus erster oder zweiter Hand beisteuern. Dies vermutlich ungeordnete Syndrom vorwiegend negativer Erinnerungen wurde durch die Berichterstattung der Medien im Jahre 1984 offensichtlich aktualisiert.

${ }^{1}$ Noch in der 10. Auflage (1966) des Handbuches ,SBZ von A bis Z“, Hrsg. Bundesministerium für gesamtdeutsche Fragen, wurde die Massen-Migration als „Abstimmung mit den Füßen“ bezeichnet (S. 147). In der 11. Auflage (1969) mit dem geänderten Titel „A bis Z“ ist diese Einschätzung am Ende des im übrigen kaum veränderten Stichwortes „Flüchtlinge“ entfallen.

${ }^{2}$ Im Mai 1984 ermittelte Allensbach, daß knapp 40\% der westdeutschen Bevölkerung die Zuwanderung aus der DDR eher sorgenvoll betrachtet, $\mathrm{da} B$ viele Menschen offensichtlich fürchten, unsere ohnehin vorhandenen Probleme könnten durch die Zuwanderer noch größer werden (Allensbacher Berichte 1984). Infratest ermittelte etwa zur gleichen Zeit ähnliche Befürchtungen in einer repräsentativen Befragung (Köhler, Ronge 1985: 52ff). Für die Daten der 50er Jahre, Noelle, Neumann (1956: 231).
Eine solche Interpretation wird durch zwei unabhängig voneinander durchgeführte demoskopische Untersuchungen nahegelegt, die im Ergebnis entsprechenden Befragungen der 50er Jahre gleichen $^{2}$.

Als Folge dieser öffentlichen Aufmerksamkeit wurden Projekte zur Herkunft der Ausgereisten und $\mathrm{zu}$ ihrer Integration in der Bundesrepublik realisiert, vergleichbar den älteren und neueren Integrationsstudien für die 50er-Jahre-Migranten.

Auffällig ist, daß alle genannten Forschungsrichtungen Quantifizierungen und Motiv-Interpretationen für die Migration der 50er Jahre verwenden. Sie bleiben hinter früher erreichten Präzisierungen und Einsichten zurück. An diesen älteren Forschungsstand soll im Folgenden erinnert werden.

\section{Die Migrations-Statistik}

Eine widerspruchsfreie und wissenschaftlich gesicherte Statistik der Migration aus der SBZ/DDR in die Bundesrepublik war 1971, fast 10 Jahre nach dem Mauerbau noch nicht verfügbar (Hardin 1971: 75). Diese Situation ist bis heute unverändert.

Die Zentralverwaltung für Statistik in der DDR hat entsprechende Zahlen nie explizit veröffentlicht; die Zahlen des Statistischen Bundesamtes haben als wesentlichen Mangel die wechselnden Definitionen für den Personenkreis „ehemalige Bewohner der SBZ/DDR“. Das einschlägige Handbuch des Bundesministers für innerdeutsche Beziehungen bietet, auch in der neuesten Auflage, nur die unzuverlässigen Zahlen des Bundesnotaufnahmeverfahrens (BMB 1985: 419). In dieser Situation erscheint es sinnvoll, die Datenquellen zum Ausmaß der Massen-Migration in den 50er Jahren knapp zu diskutieren. In Tabelle 1 sind diese Daten zusammengefaßt.

\subsection{Bevölkerungsstatistik der DDR (,DDR-Migrations-Statistik")}

Diese Migrations-Statistik basiert darauf, daß Veränderungen der DDR-Wohnbevölkerung jeweils am Ende eines Jahres, bereinigt um die „natürlichen" Bevölkerungsbewegungen (Geburt und 
Tabelle 1 Vergleich der Datenquellen: Wanderungsgewinne der Bundesrepublik 1951 bis 1961 aus der Ost-WestMigration (in Tausend)

\begin{tabular}{|c|c|c|c|c|}
\hline Jahr & $\begin{array}{l}\text { DDR-Bevölke- } \\
\text { rungs- } \\
\text { statistik }\end{array}$ & $\begin{array}{l}\text { Wanderungs- } \\
\text { statistik der } \\
\text { Bundesrepublik }\end{array}$ & $\begin{array}{l}\text { Statistik des } \\
\text { Bundesnotaufnahme- } \\
\text { verfahrens }\end{array}$ & $\begin{array}{l}\text { Volkszählung } 61 \\
\text { (Bundesrepublik) }\end{array}$ \\
\hline 1951 & 112 & 242 & 166 & 177 \\
\hline 1952 & 134 & 201 & 182 & 167 \\
\hline 1953 & 274 & 380 & 331 & 288 \\
\hline 1954 & 184 & 246 & 184 & 186 \\
\hline 1955 & 249 & 333 & 253 & 246 \\
\hline 1956 & 297 & 350 & 279 & 262 \\
\hline 1957 & 241 & 332 & 262 & 259 \\
\hline 1958 & 149 & 188 & 204 & 183 \\
\hline 1959 & 88 & 135 & 144 & 119 \\
\hline 1960 & 157 & 197 & 199 & 178 \\
\hline \multirow[t]{2}{*}{1961} & 187 & 210 & 207 & 74 (bis 5. 6. 61) \\
\hline & $2 . \overline{072}$ & $2 . \overline{814}$ & $2 . \overline{407}$ & $2 . \overline{139}$ \\
\hline Korrekturen* & +200 & -800 & --- & +100 \\
\hline Summe & $2 \overline{272}$ & $2 . \overline{014}$ & $\overline{---}$ & $2 \overline{239}$ \\
\hline
\end{tabular}

* Korrekturen: DDR-Statistik - Fortschreibungsfehler VZ 50 - VZ 64 (für 61 geschätzt).

Wanderungsstatistik - Fortschreibungsfehler VZ 50 - VZ 61 (ausschließlich als West-Ost-Migration gewertet)

Volkszählung 61 - restliche Migration zwischen dem 6. 6. 61 und dem 13. 8. 61.

Statistik des Bundesnotaufnahmeverfahrens - keine Korrekturschätzung möglich.

Quelle: Hardin (1971: 259), Table 2.5 und eigene Berechnungen

Tod) als Abwanderung in die Bundesrepublik gewertet werden (Storbeck 1964, Hardin 1971). Für die 50er Jahre ist diese Basis-Annahme für eine „DDR-Migrations-Statistik“ vermutlich gerechtfertigt. Die Statistik reflektiert die „Netto“-Veränderungen der DDR-Bevölkerung, d. h. die WestOst-Migration ist vollständig substrahiert. Der wesentliche Mangel dieser Statistik liegt im statistischen Fehler der Bevölkerungsfortschreibung, der sich bis zur Volkszählung (VZ) 1964 auf ca. 200000 ,fehlende“ Personen summiert hatte. Dieser Fehler muß nach der Logik der „DDR-Migrations-Statistik" hinzuaddiert werden (Hardin 1971: 77).

\subsection{Die Wanderungsstatistik der Bundesrepublik}

Die bei Bethlehem (1982) referierten Zahlen der Wanderungsstatistik des Statistischen Bundesamtes basieren auf dem polizeilichen Melderegister. Aufgrund der Anfälligkeit dieser Statistik für administrative Fehler (z. B. wegen der Ungenauigkeit der Angaben bei Zu- bzw. Fortzügen) ergab sich für diese Daten ein Fortschreibungsfehler für den Zeitraum 1950 bis 1961 von 750000 bis 800000 zuviel gezählten Personen (Köllmann 1983: 72, Hardin 1971: 84).
Hardin nimmt an, daß die überwiegende Mehrzahl dieser Personen ohne polizeiliche Abmeldung in die DDR abgewandert ist. Zur Begründung verweist er $u$. a. auf nicht näher nachgewiesene Daten „from interviews by West German border officials at the border between the two Germanies of persons who said they were emigrating to East Germany" (1971: 85).

\subsection{Die Statistik des Bundesnotaufnahmeverfahrens (NA V-Statistik)}

Diese Statistik reflektiert die Zahl der Anträge in den Notaufnahmelagern. Sie erfaßt also einerseits nicht alle Ost-West-Migranten und negiert andererseits die West-Ost-Migration. ,The NAV statistics are an unreliable measure of either the total or the annual east-west-migration. Their principal value is therefore qualitativ" (Hardin 1971: 79)

\subsection{Migranten-Befragung im Rahmen der VZ 1961 und der VZ 1970}

Durch die zufällige Terminierung der Volkszählung von 1961, knapp zwei Monate vor dem Mauerbau, sind in dieser Befragung weitgehend alle 
Migranten aus der DDR erfaßt und differenziert nachgewiesen. Ungenauigkeiten sind anzunehmen, da die Angaben nur dann erhoben wurden, wenn sich jemand $\mathrm{zu}$ seiner „Flüchtlingseigenschaft" bekannte.

Um den statistischen Fehler der VZ 1961 für die Migrationsstatistik (u. a. durch Antwortverweigerung oder -fälschung verursacht) abschätzen zu können, sind die mit der gleichen Methode in der VZ 1970 erhobenen Daten zum Vergleich geeignet. Die Daten stimmen sehr gut überein. Lediglich für die Geburtsjahrgänge 1946 bis 1950 wurden 1970 knapp 20\% weniger Migranten gezählt als 1961. Diese Personen sind zwischen beiden Volkszählungen volljährig geworden; waren also als Kinder von der Migration ihrer Eltern betroffen. Sie definieren sich 10 Jahre später - in einer starken Minderheit jedenfalls - nicht mehr als Migranten.

\section{Die politisch-motivierte Migration}

Die Aufgliederung der „Brutto“-Migrationszahlen (der Tabelle 1) nach Zeiträumen und späteren Status-Zuerkennungen (als „Vertriebener“ oder als „Sowjetzonenflüchtling“) präzisiert die Dimensionen der Massen-„Flucht“.

- Diese Differenzierungen sind nur für die Daten der VZ 1961 möglich: Zwischen 1950 und 1961 sind insgesamt 2.4 Mio. Migranten aus der DDR gekommen; darunter 700 Tsd., die als ,Vertriebene“ und $200 \mathrm{Tsd}$., die als „Sowjetzonenflüchtlinge“ anerkannt wurden. Dem großen „Rest“ der damaligen Migranten (1.3 Mio.) wurden in der Bundesrepublik keine politischen Migrationsgründe zugebilligt.

- Geht man davon aus, daß die Massen-Migration bis etwa 1955 von den DDR-Behörden aus innenpolitischen Gründen gewünscht bzw. sogar gewollt war (der Straftatbestand „Republikflucht" wurde erst Ende 1957 eingeführt), so umfaßt die „strittige" Migration (1955 bis 1961) 1.3 Mio. Menschen, darunter 200 Tsd. „Sowjetzonenflüchtlinge“.

Die jährliche, in der Bundesrepublik als ,politischmotivierte" anerkannte Migration aus der DDR hatte somit in den 50er Jahren eine Größenordnung, die ungefähr den gegenwärtigen jährlichen Fortzügen von Bundesbürgern in die USA entspricht.

\section{Soziologische Folgen der Migration}

Bis Mitte der 60er Jahre wurde die Migration aus der DDR vorwiegend unter allgemein-politischen Aspekten gesehen und bewertet.

Beginnend mit den Arbeiten von Storbeck (1963, 1964) setzen differenzierende Analysen über die Wirkungen der Migration für beide deutsche Staaten ein.

Für die DDR wurden vor allem Auswirkungen der Akademikerabwanderung diskutiert (Storbeck 1964, Hardin 1971, Gaus 1981), für die Bundesrepublik arbeitsmarktpolitische Effekte (Bethlehem 1982).

\subsection{Migrationsfolge: Stabilisierung der Institutionen}

Bereits 1963 hat Storbeck darauf hingewiesen, daß die Migration am Beginn der 50er Jahre (mit Einschränkungen bis 1955) für die DDR-Führung auch vorteilhaft war.

Die DDR wurde auf diesem Wege von „inaktiven“ und ,gesellschaftlich belastenden" oder nicht integrierbaren Personen entlastet. Hardin (1971) weist nach, daß im Gegensatz zum durchschnittlichen Alterstrend die Anteile der Hoch- und Fachschulabsolventen mit dem Lebensalter der Migranten anstiegen.

Eine Modellrechnung verdeutlicht, daß durch diese selegierende Wirkung der Migration der Anteil systemkonformer, loyaler Personen in Leitungspositionen zwischen 1950 und 1961 sich verdoppelt hat.

\subsection{Migrationsfolge: Entlastung des Arbeitsmarktes}

Bethlehem (1982: 81ff) hat die Wirkung der Migration für die Bundesrepublik vor allem im zuströmenden Arbeitskräftepotential gesehen.

Zunächst, am Beginn der 50er Jahre, diskutierte der Bundestag noch Regelungen, um die Migration aus der DDR zu begrenzen. In der bald danach beginnenden wirtschaftlichen Aufschwungphase war die Migration jedoch sehr willkommen und die im Bundesnotaufnahmegesetz vorgesehenen Restriktionen wurden nicht angewandt. Dafür waren natürlich neben den ökonomischen Motiven vor allem politische maßgebend.

Die arbeitsmarktpolitische Bedeutung der Migration belegt Bethlehem u. a. mit der notwendigen 
Forcierung der Gastarbeiteranwerbung in den Jahren nach dem Mauerbau.

\subsection{Die Migrationsfolge: „Staatsvolk der kleinen Leute“"}

In seinem Buch „Wo Deutschland liegt" hat Gaus (1983) die Sozialstruktur der DDR mit der griffigen Formel „Staatsvolk der kleinen Leute“ bezeichnet.

Die behauptete selektive Wirkung der Migration in den 50er Jahren (,nur die kleinen Leute sind zurückgeblieben“) ist als sozio-demographisches Faktum durchaus nachweisbar. Gaus' Erklärung, daß das geistige und gesellschaftliche „kleine Leute"-Klima in der DDR durch die Abtrennung, die Abwanderung der tonangebenden Ober- und oberen Mittelschicht verursacht sei, ist jedoch zu mechanisch gedacht.

Als Erklärungen sind sozial-historisch orientierte Modelle eher geeignet, wie sie z. B. von Elias (1976) entwickelt wurden. Als Konsequenz eines Elitenwechsels ,durchdringen sich Verhaltensweisen der jeweils oberen und der aufsteigenden unteren, Schichten. . . (Elias 1976: 421).

Die auffällige von Gaus erstmals beschriebene Gleichzeitigkeit von irritierenden Rudimenten bürgerlich-deutscher Tradition neben bürokratisch-unionistischen Verkehrsformen, gepaart mit stalinistisch-zentralistischer Schwerfälligkeit und Kontrolle wäre, folgt man Elias, aus der „Abkunft" der DDR zu erklären: Ein deutscher Staat mit Wurzeln in der (preußischen) Arbeiterbewegung, eingerichtet und gestützt durch die Sowjetunion.

\section{Individuelle Migrations-Gründe}

Die individuellen Gründe der Migranten zum Verlassen der DDR sind - besonders in den 50er Jahren - häufig interpretiert und politisch gewertet worden (u. a. Storbeck 1963).

Repräsentative Befragungen, zuverlässige Befunde über die Motive für die Migration sind bis heute nicht vorhanden.
Beim Wechsel in die Bundesrepublik wurden jedoch fast alle ehemaligen DDR-Bürger von deutschen und alliierten Dienststellen ausführlich befragt. Die erstellten Akten sind - aufgrund von Zufällen - zumeist erhalten geblieben, sind jedoch noch immer klassifiziert und unzugänglich.

Dieses Material wäre hervorragend geeignet, die individuellen Aspekte der Ost-West-Migration aufzuhellen.

Die Lebensdaten und Lebensumstände von ehemaligen Mitgliedern in DDR-Parteien z. B., die durch Befragungen dieser Personen in den „OstBüros" der jeweils entsprechenden Partei in der Bundesrepublik erhoben wurden, wären wichtige Dokumente für die zeitgeschichtlich-soziologische Forschung.

\section{Literatur}

Bade, K. J., 1983: Vom Auswanderungsland zum Einwanderungsland. Berlin: Colloquium.

Bethlehem, S., 1982: Heimatvertreibung, DDR-Flucht, Gastarbeiterzuwanderung. Stuttgart: Kohlhammer.

Bundesministerium für gesamtdeutsche Fragen (Hrsg.), 1966: SBZ von A bis Z. Bonn: Deutscher BundesVerlag.

ders., 1969: A bis Z. Bonn: Deutscher Bundes-Verlag.

Elias, N., 1976: Über den Prozeß der Zivilisation, 2 Bd. Frankfurt/M.: Suhrkamp.

Gaus, G., 1983: Wo Deutschland liegt. Hamburg: Hoffmann und Campe.

Hardin, W. R., 1971: Emigration, occupational mobility and institutionalization. The German Democratic Republic. Cambridge, Mass.: MIT (Microfilm).

Köhler, A., Ronge, V., 1985: Ein Test auf Wiedervereinigung. Deutschland Archiv 18: 52-59.

Köllmann, W., 1983: Die Bevölkerungsentwicklung der Bundesrepublik, in: W. Conze, W. R. Lepsius (Hrsg.), Sozialgeschichte der Bundesrepublik Deutschland. Stuttgart: Kohlhammer.

Marschalck, P., 1984: Bevölkerungsgeschichte im 19. und 20. Jahrhundert. Frankfurt/M.: Suhrkamp.

Noelle, E., Neumann, P. (Hrsg.), 1956: Jahrbuch der öffentlichen Meinung 1947-1955. Allensbach: Verlag für Demoskopie.

Storbeck, D., 1963: Flucht oder Wanderung? Soziale Welt 14: 153-171

ders., 1964: Soziale Strukturen in Mitteldeutschland. Berlin: Duncker \& Humblot. 